\title{
Extended description of tunnel junctions for distributed modeling of concentrator multi-junction solar cells
}

\author{
P. Espinet , I. García, I. Rey-Stolle, C. Algora, M. Baudrit
}

\begin{abstract}
A B S T R A C T
One of the key components of highly efficient multi-junction concentrator solar cells is the tunnel junction interconnection. In this paper, an improved 3D distributed model is presented that considers real operation regimes in a tunnel junction. This advanced model is able to accurately simulate the operation of the solar cell at high concentrations at which the photogenerated current surpasses the peak current of the tunnel junction. Simulations of dual-junction solar cells were carried out with the improved model to illustrate its capabilities and the results have been correlated with experimental data reported in the literature. These simulations show that, under certain circumstances, the solar cell's short circuit current may be slightly higher than the tunnel junction peak current without showing the characteristic dip in the $J-V$ curve. This behavior is caused by the lateral current spreading toward dark regions, which occurs through the anode/p-barrier of the tunnel junction.
\end{abstract}

\section{Introduction}

High concentration photovoltaic (PV) systems seem to be one of the most promising ways to generate electricity at competitive prices for terrestrial applications [1]. In recent years, the efficiency of multi-junction solar cells (MJSCs) has increased considerably [2-4]. One of the key advances that has led to the creation of these high efficiency multi-junction solar cells is the development of a low-loss interconnection system which minimizes the optical and electrical losses in the second or third subcells. A thin, highly doped tunnel junction (TJ) is the ideal choice for connecting the cells monolithically. However, when the current density of the solar cell increases beyond the maximum tunneling current density of the tunnel junction (i.e., its peak current density, $J_{p}$ ), a dip appears in the $J-V$ characteristic curve, which reduces the conversion efficiency [5]. Therefore, so far solar cell manufacturers have tried to ensure that the solar cell is within the tunneling region when it operates around its nominal concentration level. However, it has been recently reported in [6] that there is a dependence of the current density at which the dip in the $J-V$ curve occurs with the degree of localized light intensity. In this respect, simulation models are fundamental tools for increasing the understanding of solar cells containing tunnel junctions. On one hand, 3D distributed models based on elemental circuit units are an excellent tool to represent the device under a wide range of concentration conditions $[7,8]$. However, to date and to the best of our knowledge, the tunnel junction has been simplistically modeled as a resistor [8] or even as a short circuit [9], which only takes the tunneling region into account. This simplified model is not accurate at high current densities, and it fails in situations that produce in the solar cell current densities higher than the peak current density of the tunnel junction, as reported in [6]. On the other hand, numerical simulations are based on the discretization and integration of the set of coupled differential equations which describe the solar cell. There is scarce literature on the use of them for modeling tunnel junctions in MJSCs $[10,11]$. However, numerical models are significantly more time consuming [8] and satisfactory results have yet only been shown in 2D studies of dual-junctions [10,11]. Their 3D development is still ongoing at present. In order to address the previously described limitations of both models, this paper presents a more precise distributed model that operates without restriction throughout the entire $J-V$ curve of the tunnel junction. In this improved model, the tunnel junction is modeled using an analytical expression that reproduces the three operating regimes of the tunnel junction and the lateral current spreading along the TJ layers by means of resistors. The model's potential is demonstrated in this paper by applying it to the analysis of certain experimental illustrative cases. For example, the peculiarities in the $J-V$ observed in solar cells with tunnel junctions (dip) are accurately reproduced [5]. The apparent dependence of the $J_{p}$ with the localized light intensity experimentally observed in [6] is explained through simulations with the advanced model, due to a spreading of current along the layers of the tunnel junction. This result is also consistent with numerical simulations of dual-junction solar cells presented in [12]. 


\section{Description of the 3D distributed circuit model}

The philosophy of the 3D distributed model presented in this paper has been explained in detail elsewhere [7]. This approach consists in dividing the solar cell into elementary units and assigning a suitable circuit model to each unit, depending on its geometry and position in the solar cell area (shadowed, exposed, or perimeter). The complete solar cell can subsequently be modeled by an electrical circuit that is obtained by interconnecting every unit-circuit with its neighbors. The resulting equivalent circuit formed by thousands of elements is resolved by using the SPICE circuit simulator software package. The circuits that describe the various solar cell regions are depicted in Fig. 1.

The key new aspects of the improved model are the following: (a) the tunnel diode definition, which includes a full description of its $J-V$ characteristics; and (b) the addition of lateral resistors surrounding the tunnel junction ( $r_{\text {anode }}$ and $r_{\text {cathode }}$ in Fig. 1 ). The $J-V$ curve of the tunnel junction (see Fig. 2) can be introduced either as a table with values that are either expected or measured, or as an analytical expression $J_{T J}=J_{T J}(V)$. In this paper, the complete static $J-V$ characteristic of the tunnel diode junction was modeled using the following analytical expressions [13]:

$J_{T J}=J_{t}+J_{x}+J_{t h}$

$J_{t}=J_{p}\left(V / V_{p}\right) \exp \left(1-V / V_{p}\right)$

$J_{x}=J_{v} \cdot \exp \left[A\left(V-V_{v}\right)\right]$

$J_{t h}=J_{0}[\exp (q V / k T)-1]$
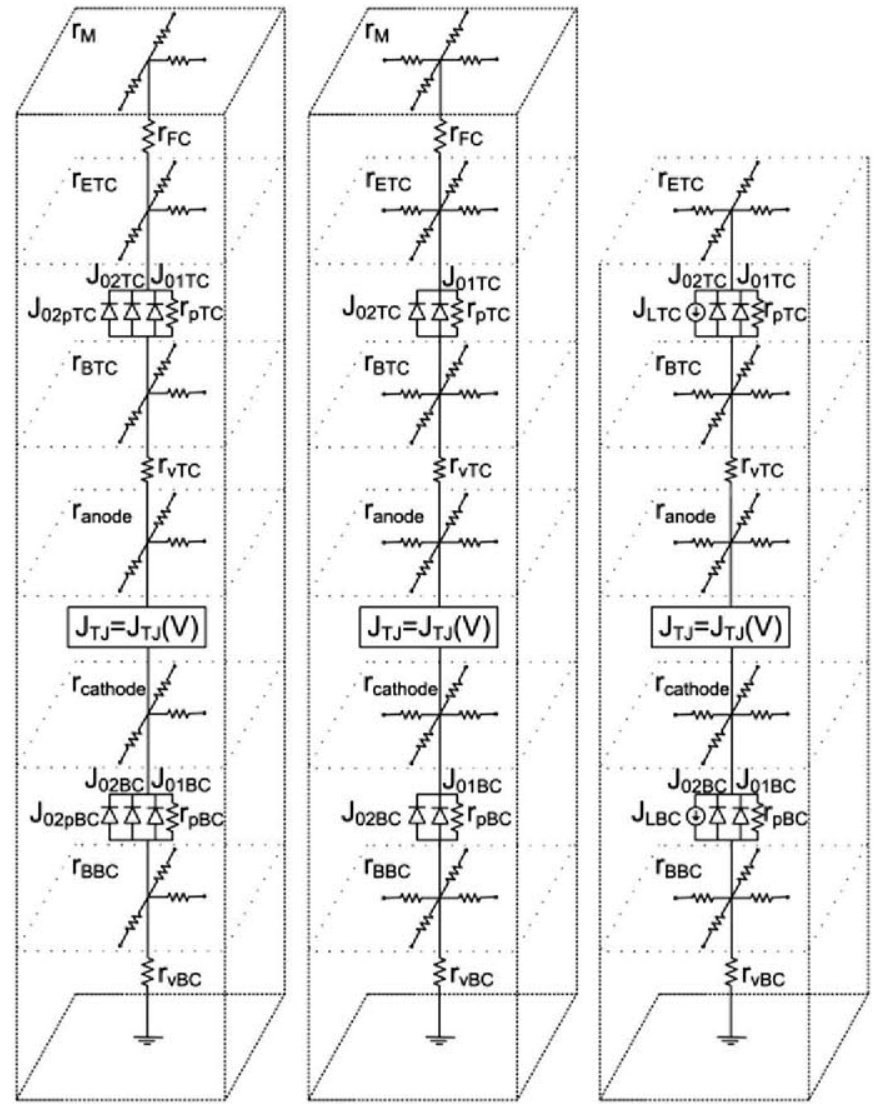

Fig. 1. From left to right, the elementary unit cell of a perimeter area, dark area and illuminated area of a dual-junction solar cell are presented. In this advanced model, the tunnel junction is modeled with a component represented by an analytical expression and lateral resistances ( $r_{\text {anode }}$ and $\left.r_{\text {cathode }}\right)$. A detailed description of the components can be found elsewhere [7].

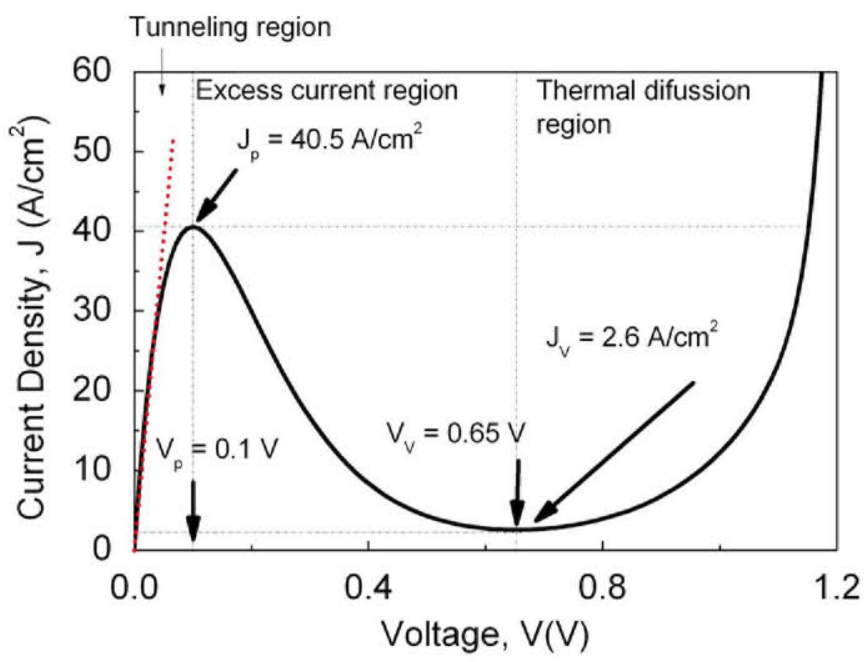

Fig. 2. $J-V$ characteristic of the tunnel junction used in the simulations presented in this paper. The red dotted line represents the resistor used in previous simulations to model the tunneling region. Only the named ohmic region has been taken into account in previous reports but, as shown in the figure, the simplified model is only accurate in the case of low and moderate currents. The characteristic parameters of the $J-V$ curve appear as labels in the figure: peak current density $\left(J_{p}\right)$, peak voltage $\left(V_{p}\right)$, valley current density $\left(J_{V}\right)$ and valley voltage $\left(V_{V}\right)$. (For interpretation of the references to color in this figure legend, the reader is referred to the web version of this article.)

where $J_{T J}$ is the current density of the tunnel junction $\left(\mathrm{A} / \mathrm{cm}^{2}\right) ; J_{t}$ is the tunneling current density $\left(\mathrm{A} / \mathrm{cm}^{2}\right) ; J_{x}$ is the excess current density $\left(\mathrm{A} / \mathrm{cm}^{2}\right) ; J_{\text {th }}$ is the thermal current density $\left(\mathrm{A} / \mathrm{cm}^{2}\right) ; J_{p}$ is the peak current density $\left(\mathrm{A} / \mathrm{cm}^{2}\right) ; V_{p}$ is the peak voltage $(\mathrm{V}) ; J_{v}$ is the valley current density $\left(\mathrm{A} / \mathrm{cm}^{2}\right) ; V_{v}$ is the valley voltage $(\mathrm{V})$; $A$ is a constant $\left(\mathrm{V}^{-1}\right) ; J_{0}$ is the recombination current density $\left(\mathrm{A} / \mathrm{cm}^{2}\right)$; and the other parameters have the standard meaning.

\section{Simulation procedure}

For the sake of clarity, a dual-junction GaInP/GaAs solar cell was used as the test vehicle for the simulations. This device has a single tunnel junction; thus, the implications are clearer than for a triple junction and the potential of the model is more directly apparent. The dual-junction solar cell characteristics used in this paper's analysis are based on a specific device [2]. Briefly, the device is a high concentrator $\mathrm{GaInP} / \mathrm{GaAs}$ solar cell with an active area of $1 \mathrm{~mm}^{2}$ and a front metal grid featuring an inverted square configuration that is comprised of eight evenly spaced fingers that are $3 \mu \mathrm{m}$ wide, producing a shadowing factor (FS) of $2.7 \%$, surrounded by a busbar that is $100 \mu \mathrm{m}$ wide (see details in [2]). In order to simplify the interpretation of the simulations presented in this paper, it is assumed that both subcells are current matched at a short circuit current density of $13.5 \mathrm{~mA} / \mathrm{cm}^{2}$ at 1 sun (AM1.5D low AOD, $1000 \mathrm{~W} / \mathrm{m}^{2}$ ). The $J-V$ characteristic of the tunnel junction used in the simulations is depicted in Fig. 2. We decided not to use the real tunnel junction of these devices (based on AlGaAs/GaAs heterostructure) [2] because its peak tunneling current is so high that its effects become negligible for concentrations below 20,000 suns. Instead, we decided to use a tunnel junction more similar to that found in state-of-the-art MJSC, such as the one depicted in Fig. 2 . It should be noted that the value of $J_{p}$ in this tunnel junction $\left(40.5 \mathrm{~A} / \mathrm{cm}^{2}\right)$ is equivalent to an irradiance of $3000 \mathrm{X}$ in our test device. In summary, the simulated device includes the top and bottom cell features described previously [2], together with the tunnel junction sketched in Fig. 2. In order to obtain precise simulations, the number of nodes used in the 
simulations is around 90,000 and the number of components is approximately 125,000 .

\section{Simulation results and experimental evidence previously reported}

In a two-terminal multi-junction device, it is not possible to measure the $J-V$ curve independently of the tunnel junction. However, a widely used method to determine the peak current of the tunnel diode in the multi-junction stack is to increase the light intensity on the solar cell and monitor the $J$-Vcurve until the dip in the curve appears [5]. At this point, it can be assumed that the short circuit current density of the solar cell $\left(J_{s c}\right)$ equals the tunnel diode peak current density $\left(J_{p}\right)$. However, it has been recently reported in [6] that there is a dependence of $J_{p}$ with the level of localized light intensity. Moreover, in solar cells with a non-uniform metalization grid, it has been observed in [6] that at a fixed localized light intensity, the $J_{p}$ varies spatially. Therefore, in both cases the variation of the $J_{p}$ is related to the solar cell's dark area. In other words, the larger the dark area of the solar cell (smaller localized light intensity or higher shadowing factor, FS), the higher the concentration at which the dip in the $J$-Vcurve comes out. As any solar cell naturally leads to a lack of uniformity due to the existence of illuminated and dark areas, we have reproduced the phenomena reported in [6] by simulating dualjunction solar cells, all of them with the tunnel junction sketched in Fig. 2, with different grids. The front grids compared have (a) eight evenly spaced fingers $2 \mu \mathrm{m}, \mathrm{FS}=1.8 \%$, (b) eight evenly spaced fingers $3 \mu \mathrm{m}$ wide, $\mathrm{FS}=2.7 \%$, and (c) eight evenly spaced fingers $5 \mu \mathrm{m}, \mathrm{FS}=4.4 \%$. Fig. 3 represents the simulated $J-V$ curves

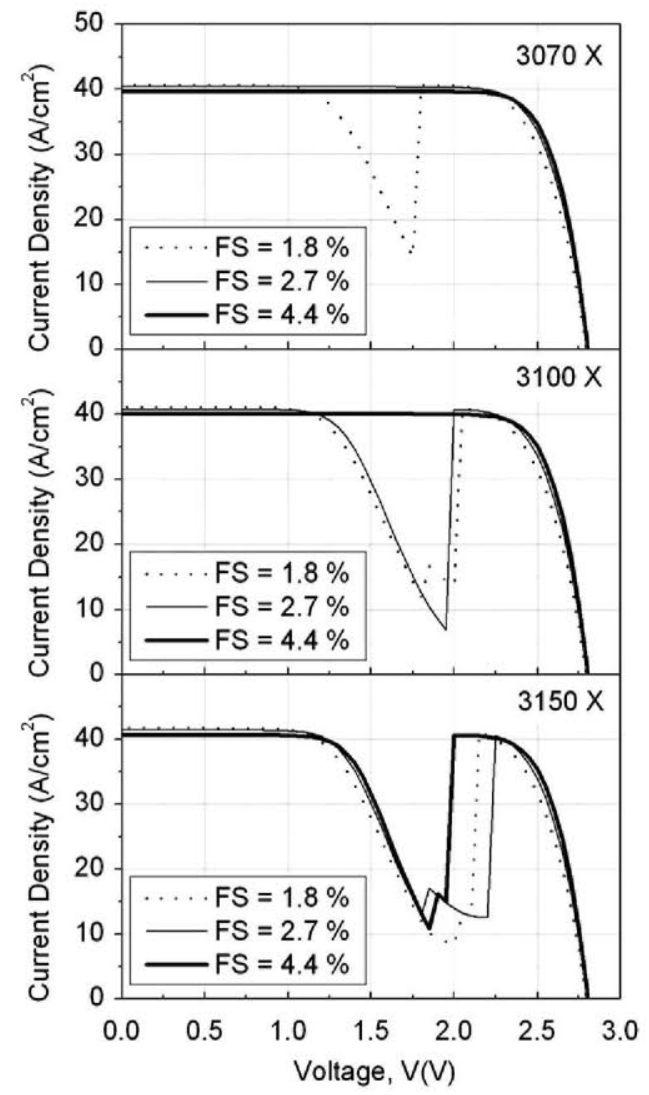

Fig. 3. $J$ - $V$ curves of dual-junction solar cells with three different FS: $1.8 \%$ (dots), $2.7 \%$ (thin line) and $4.4 \%$ (thick line), under the three concentrations at which the dip in the $J-V$ curve appears for each grid, $3070 \times(F S=1.8 \%), 3100 \times$ (FS $=2.7 \%$ ) and $3150 \times(\mathrm{FS}=4.4 \%)$

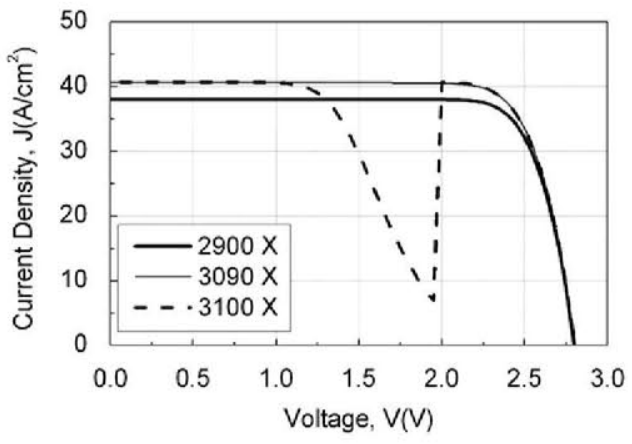

Fig. 4. $J-V$ curves for a dual-junction solar cell [2] with the tunnel junction in Fig. 2 under uniform irradiation conditions at different concentration levels.

of the three different grids at the three significant concentrations: (1) $3070 \times$ the concentration at which the dip in the $J-V$ curve of the solar cell with $\mathrm{FS}=1.8 \%$ appears, (2) $3100 \mathrm{X}$ the concentration at which the dip in the $J-V$ curve of the solar cell with $\mathrm{FS}=2.7 \%$ is observed, and (3) $3150 \times$ the concentration at which the dip in the $J-V$ curve of the solar cell with $\mathrm{FS}=4.4 \%$ occurs. Therefore, Fig. 3 shows that in all the simulated cases the dip in the $J-V$ curve appears at a concentration higher than $J_{p}$ which occurs at $3000 \mathrm{X}$. Also, the same tendency as the one reported in [6] is observed, the higher the FS (higher metalization cover, consequently higher the dark area) the higher the concentration at which the dip in the $J-V$ curve occurs.

In order to better understand the result obtained in the simulations, the case of the grid with fingers $3 \mu \mathrm{m}$ wide (case b, $\mathrm{FS}=2.7 \%$ ) has been studied in detail. Fig. 4 depicts the results of the simulations at different concentration levels: $2900 \mathrm{X}$, which represents a concentration lower than the supposed peak of the tunnel junction $(3000 \mathrm{X})$; and two concentrations that are slightly higher than the expected peak of the tunnel junction, namely $3090 \mathrm{X}$ and $3100 \mathrm{X}$. As expected, at a concentration lower than $3000 \mathrm{X}$, no effects are apparent in the simulated $J-V$ curve; while at higher concentrations (i.e., at $3100 \mathrm{X}$ ) the expected dip in the $J-V$ curve becomes apparent. However, surprising results are obtained at concentrations slightly above $3000 \mathrm{X}$ : at $3090 \mathrm{X}$, no effects in the $J-V$ are detectable. Therefore, from Fig. 4, it can be inferred that the dual-junction device can operate with illumination currents beyond the tunnel diode peak current without exhibiting the dramatic dip-related effects, at least up to approximately $3090 \mathrm{X}$. In order to understand the origin of these features a detailed analysis of these simulations has been done in Fig. 5 . This figure shows that when the current in the solar cell exceeds the peak current of the tunnel junction, the excess current flows laterally until it reaches a dark area (beneath a finger or busbar) where it is able to flow vertically. In other words, dark areas represent an 'unused' tunnel junction area that can contribute to sink some photocurrent if lateral current spreads toward those dark areas (when the lateral resistances are not elevated). A direct consequence of this interpretation is that the extra short circuit current that the solar cell can handle before the dip occurs is directly proportional to the shadowed area accessible to the current spreading, which is typically close to the area covered by the grid. Essentially what happens in our simulation is the maximum short circuit current without a dip (occurring at 3090 $\mathrm{X}$ ) is approximately $3 \%$ higher than the tunnel junction peak current, as a result of the $2.7 \%$ shadowing factor (FS) in our design plus a minor $(\approx 0.3 \%)$ current spreading under the busbar. Current spreading across the tunnel junction and adjacent layers toward dark regions was also suggested in Ref. [6] as a plausible reason for the variations observed in $J_{p}$. Also, the same phenomena, a non-negligible current spreading through the layers of the 
$3090 \mathrm{X}$

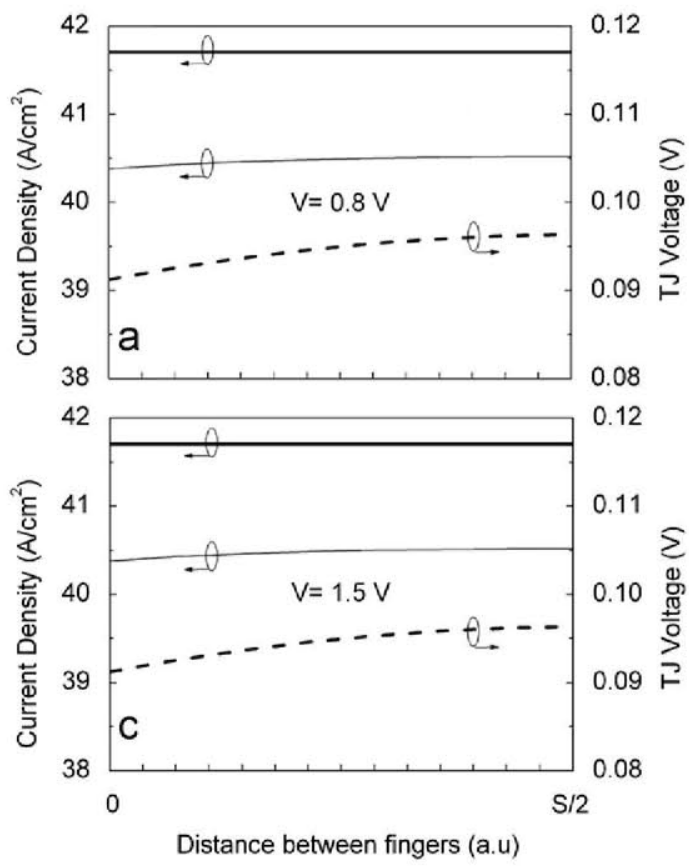

$3100 x$

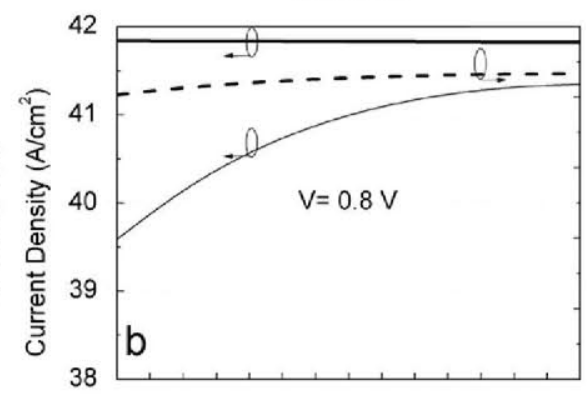

1.16

1.15

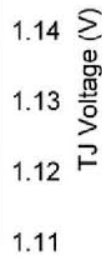

1.14

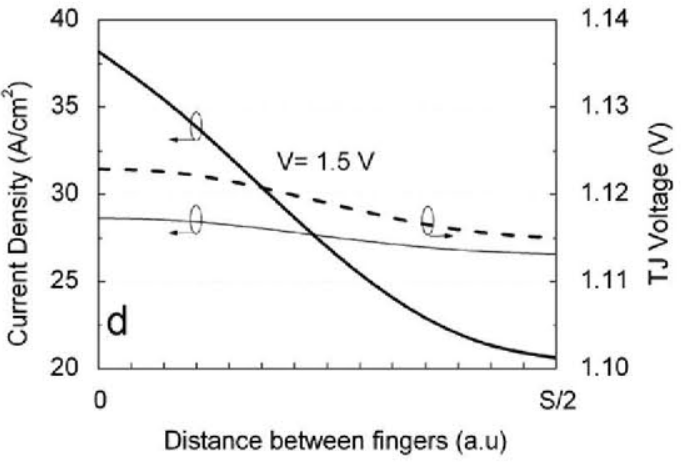

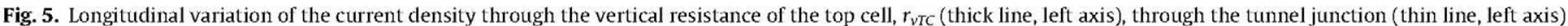

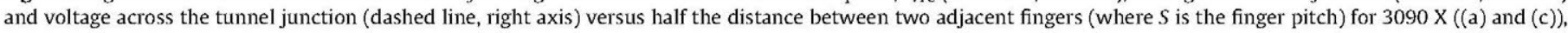
for $3100 \mathrm{X}((\mathrm{b})$ and $(\mathrm{d}))$, and for two different dual-junction cell voltages: $0.8 \mathrm{~V}$ and $1.5 \mathrm{~V}$.

tunnel junction has been observed in simulations of dual-junction solar cells with numerical simulation models in [12] (often referred as technology computer-aided design, TCAD).

Fig. 5 aids in quantifying the current spreading that occurs in the dual-junction solar cell used in Fig. 4. The spatial variation along half the distance between two adjacent fingers, of the main currents and voltages around the tunnel junction are plotted for two concentrations: $3090 \times$ (no dip) and $3100 \times$ (with dip). If the current density through the vertical resistance in the top cell is larger than the current through the tunnel junction, it means that the excess of current from the top cell has flown away trough the anode resistances $\left(r_{\text {anode }}\right)$. If instead the current density through the tunnel junction is larger than the current through the top cell, then there has been an injection of current from the anode resistances to the tunnel junction. In other words, this point is draining the excess of current photogenerated in other regions.

For each concentration, two solar cell voltages are considered: (a) $0.8 \mathrm{~V}$ before the dip occurs and (b) $1.5 \mathrm{~V}$ in the dip. At $3090 \mathrm{X}$ and at low voltages (Fig. 5(a) and (c)), the tunnel junction operates in the tunneling region at the two voltages analyzed. In this case, current density through the tunnel junction (thin line) is smaller than its peak current $\left(40.5 \mathrm{~A} / \mathrm{cm}^{2}\right)$ and corresponding voltages (dashed line) are also smaller than its peak voltage $(100 \mathrm{mV})$. This phenomenon is possible, despite the fact that the photogenerated current density $\left(41.7 \mathrm{~A} / \mathrm{cm}^{2}\right)$ is higher than the TJ peak current density $\left(40.5 \mathrm{~A} / \mathrm{cm}^{2}\right)$, because there exists a lateral current density through $r_{\text {anode }}$ (Fig. 1) which diverts the excess current toward the dark areas (the current density through the vertical resistance in the top cell is higher than the current density through the tunnel junction along the whole finger pitch). Consequently, $r_{\text {anode }}$ is a key parameter on the appearance of the dip in the $J-V$ curve. A higher value of $r_{\text {anode }}$ would be translated in a smaller current spreading, then, the dip in the $J-V$ curve would appear at a lower concentration, and vice versa. In summary, in Fig. 5(a) and (c), we used the tunnel junction at its 'maximum capacity' within the tunneling region, both within the illuminated and dark areas. Accordingly, we see no dip in the $J-V$ curve.

Slightly increasing the concentration to $3100 \mathrm{X}$ dramatically changes this situation. At $0.8 \mathrm{~V}$ (Fig. 5(b)), the tunnel junction voltage is around $1.15 \mathrm{~V}$, which indicates that the tunnel junction is now working in the thermal diffusion regime (Fig. 2). At this voltage, the current through the tunnel junction exceeds the peak current in the illuminated area away from the fingers. At $1.5 \mathrm{~V}$ and $3100 \times$ (Fig. 5(d)), where the cell is operating in the dip region, the tunnel junction is in the thermal diffusion region, but the voltage drop across it decreases to a voltage around $1.12 \mathrm{~V}$; its current therefore decreases sharply (Fig. 2), causing the dip in the $J-V$ curve.

\section{Conclusions}

This paper reports a distributed simulation model for multijunction concentrator solar cells in which the tunnel junction is fully described. The model is based on dividing the solar cell into elementary units and assigning each unit a suitable circuit. Interconnecting every unit-circuit with its neighbors yields the equivalent circuit of the whole solar cell, which can be solved using SPICE. The key new aspects of the improved model are as follows: (a) a tunnel diode definition using an analytical expression that accounts for its three operational states (tunneling, excess current and thermal diffusion); and (b) the addition of lateral resistors surrounding the tunnel junction. To assess the potential of the model, simulations were performed and correlation with experimental data was undertaken. These simulations suggest that, under certain circumstances, the solar cell short circuit current may be slightly higher than the tunnel junction peak current without exhibiting the characteristic dip in the $J-V$ curve. This result is consistent with both experimental 
measurements and with numerical simulations that have been reported elsewhere. The reason for this behavior is that the lateral current spreads toward dark regions, where some 'unused' tunnel junction area is still available. In this sense, the simulations show that the dip in the $J$-Vcurve only occurs when such current spreading is unable to maintain the whole tunnel junction below its peak current. Because this spreading occurs through the anode of the tunnel junction, its equivalent lateral resistance $\left(r_{\text {anode }}\right)$ is the key parameter that influences the appearance of the dip in the $J-V$ curve.

\section{Acknowledgments}

This paper was supported by the Spanish Ministerio de Educación y Ciencia under the CONSOLIDER-INGENIO 2010 program by means of the GENESIS FV project (CSD2006-004) and the research projects with references TEC2007-29630, TEC200801226 and PSE-440000-2009-8. The Comunidad de Madrid has also contributed under the NUMANCIA Program (S2009/ENE/477).

\section{References}

[1] A. Luque, G. Sala, I. Luque-Heredia, Photovoltaic concentration at the onset of its commercial deployment, Prog. Photovolt. Res. Appl. 14 (5) (2006) $413-428$.

[2] I. Garcia, I. Rey-Stolle, B. Galiand, C. Algora, A 32.6\% efficient lattice-matched dual-junction solar cell working at 1000 suns, Appl. Phys. Lett. 94 (5) (2009) 053509 ( 3 pp.)

[3] R.R. King, A. Boca, W. Hang, L. X-Q, D. Bhusari, D. Larrabee, K. Edmondson, D. Law, C. Fetzer, S. Messopian, N. Karam, Band-gap-engineered architectures for high-efficiency multijunction concentrator solar cells, in: Twenty-fourth
European Photovoltaic Solar Energy Conference and Exhibition, Hamburg, Germany, 21-25 September, 2009, pp. 55-61.

[4] S. Wojtczuk, P. Chiu, X. Zhang, D. Derkacs, C. Harris, D. Pulver, M. Timmons, InGaP/GaAs/lnGaAs 41\% concentrator cells using bi-facial epigrowth, in: 35th IEEE Photovoltaic Specialists Conference (PVSC)2010, pp. 001259-001264. doi:10.1109/PVSC.2010.5614196.

[5] V. Andreev, E. Ionova, V. Larionov, V. Rumyantsev, M. Shvarts, G. Glenn, Tunnel Diode Revealing Peculiarities at I-V Measurements in Multijunction III-V Solar Cells, in: Conference Record of the 2006 IEEE 4th World Conference on Photovoltaic Energy Conversion, vol. 1, 2006, pp. 799-802, doi:10.1109/WCPEC.2006.279577.

[6] A. Braun, B. Hirsch, E.A. Katz, J.M. Gordon, W. Guter, A.W. Bett, Localized irradiation effects on tunnel diode transitions in multi-junction concentrator solar cells, Sol. Energy Mater. Sol. Cells 93 (9) (2009) 1692-1695.

[7] B. Galiana, C. Algora, I. Rey-Stolle, I.G. Vara, A 3-D model for concentrator solar cells based on distributed circuit units, IEEE Trans. Electron Devices 52 (12) (2005) 2552-2558.

[8] I. Rey-Stolle, C. Algora, I. Garcia, M. Baudrit, P. Espinet, B. Galiana, E. Barrigon, Simulating III-V concentrator solar cells: a comparison of advantages and limitations of lumped analytical models, distributed analytical models and numerical simulations, in: 35th IEEE Photovoltaic Specialist Conference, IEEE Philadelphia USA, 2009, pp. 001622-001627.

[9] K. Nishioka, T. Takamoto, W. Nakajima, T. Agui, M. Kaneiwa, Y. Uraoka, T. Fuyuki, Analysis of triple-junction solar cell under concentration by spice, in: Proceedings of 3rd World Conference on Photovoltaic Energy Conversion, vol. 1, 2003, pp. 869-872.

[10] M. Baudrit, C. Algora, Tunnel diode modeling, including nonlocal trapassisted tunneling: a focus on III-V multijunction solar cell simulation, IEEE Trans. Electron Devices 57 (10) (2010) 2564-2571. doi:10.1109/ TED.2010.2061771.

[11] M. Hermle, G. Lètay, S.P. Philipps, A.W. Bett, Numerical simulation of tunnel diodes for multi-junction solar cells, Prog. Photovolt. Res. Appl. 16 (5) (2008) 409-418 http://dx.doi.org/10.1002/pip.824.

[12] J.M. Olson, Simulation of nonuniform irradiance in multijunction IIIV solar cells, in: 35th IEEE Photovoltaic Specialists Conference (PVSC)2010, pp. 000201-000204 doi:10.1109/PVSC.2010.5614523.

[13] S.M. Sze, Physics of Semiconductor Devices, second ed., Wiley, New York, $1981,523 \mathrm{pp}$. 